\title{
Trend Analysis of HIV Testing Services in Zimbabwe, 2007-2016: A Secondary Dataset Analysis
}

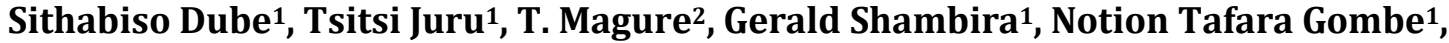 \\ Mufuta Tshimanga ${ }^{1}$
}

${ }^{1}$ Department of Community Medicine, University of Zimbabwe, Harare, Zimbabwe

${ }^{2}$ National AIDS Council, Harare, Zimbabwe

Email: tjuru@zimfetp.net

How to cite this paper: Dube, S., Juru, T., Magure, T., Shambira, G., Gombe, N.T. and Tshimanga, M. (2017) Trend Analysis of HIV Testing Services in Zimbabwe, 2007-2016: A Secondary Dataset Analysis. Open Journal of Epidemiology, 7, 285-297. https://doi.org/10.4236/ojepi.2017.73023

Received: April 24, 2017

Accepted: August 26, 2017

Published: August 29, 2017

Copyright $\odot 2017$ by authors and Scientific Research Publishing Inc. This work is licensed under the Creative Commons Attribution International License (CC BY 4.0).

http://creativecommons.org/licenses/by/4.0/

\section{Abstract}

Background: HIV Testing Services (HTS) is a full range of services (e.g. counselling and post-test referrals) that are offered together with HIV testing. It is an important prevention strategy and the gateway to treatment. The national targets in 2016 were to test 1.1 million people of which $54 \%$ was achieved. We determined trends of HTS in Zimbabwe from 2007 to 2016. Methods: A secondary dataset analysis was conducted using National Aids Council CoreOutput Indicators dataset. Variables captured include total and repeat tests, counselling and referrals. Microsoft excel and Epi Info was used to generate frequencies, percentages and conduct chi square test for trends. Panda-Class Libraries was to attain estimates of HTS uptake till 2020. We used $\chi^{2}$ and pvalues for statistical significance. Results: All $(10,847.223)$ records were analysed. HIV tests per year increased from 340,705 in 2007, to 1,974,795 in 2015 ( $\chi^{2}$ 0.10492, $\mathrm{p}$-value 0.74615$)$. In $2007,31 \%(\mathrm{n}=106,884)$ clients tested positive whilst in 2016 only $7 \%(\mathrm{n}=121,196)$ were positive $\left(\chi^{2} 0.01166\right.$, $\mathrm{p}$-value $0.91402)$. The $25-49$ year age-group tested consistently highest throughout the 10year period ( $\chi^{2} 0.0558 \mathrm{p}$-value 0.813 ). The $15-24$ year age-group had the highest yield ( $11 \%$ in 2015$)$. Females ( $\chi^{2} 0.1074$, p-value 0.743$)$ consistently tested higher than males ( $\chi^{2} 0.0614, \mathrm{p}$-value 0.804$)$. From 2007 to 2013 women had higher yields but by September 2016 males had a higher positivity of $8 \%$ (p-value $<0.05$ ). Repeat tests increased exponentially from $9 \%$ in 2012 to $78 \%$ in 2016 . Significantly more individuals $(1,924,410: 2015)$ tested than couples $(227,868: 2015)$ but couples consistently had a higher yield $\left(\chi^{2} 0.658\right.$ p-value $=0.417)$. We estimate that 179,935 people living with HIV will know their status by 2020. Conclusion: HIV tests in Zimbabwe have increased but yield has decreased. Increase in repeat tests may be an indication of exhaus- 
tion of particular HTS strategies. Following this analysis it was recommended that HTS utilize various models such as HIV self-test to cater for populations with high yields.

\section{Keywords}

HIV Testing Services, Secondary Dataset

\section{Introduction}

HIV testing is an integral component of HIV prevention strategies and provides a gateway to treatment and care. Early knowledge of HIV status is important for linking those living with HIV to timely medical care and those HIV-negative to prevention services [1]. HIV treatment is regarded a form of prevention because early provision of antiretroviral therapy (ART) has been shown to reduce HIV transmission [2]. Over the past decade, as the expansion of ART in the developing world has made medications and treatment more widely available, HIV testing and efforts to link HIV-positive individuals to available treatment and care have become important policy priorities. Even so, lack of knowledge of HIV status remains an important programmatic barrier to initiating ART support [3].

The term HIV Testing Services (HTS) is used to indicate the full range of services that a client, couple, or family is offered together with HIV testing. This includes pre and post-test counselling; linkage to appropriate HIV prevention, care and treatment services and other clinical support services; and coordination with laboratory services to support quality assurance and delivery of correct results. Although many different approaches exist for administering HTS, in general, the intervention consists of five core components: pre-test counseling that outlines the testing process, a risk-behavior assessment, informed consent of each participant, administration of the HIV test and post-test counseling based on the test result(s) [4] [5].

The World Health Organization (WHO), Joint United Nations Programme on HIV/AIDS (UNAIDS), and many other organizations have endorsed the concept of "universal access" to knowledge of HIV status. They have also endorsed the concepts of universal access to HIV prevention, treatment, care, and support, as well as protection from discrimination based on HIV-positive status. This stance is articulated in UNAIDS's vision of: zero new HIV infections, zero discrimination, and zero AIDS-related deaths and the Global Health Sector Strategy on HIV/AIDS 2011-2015 [6]. The 2012 WHO Strategic HTC Programme Framework describes a set of minimum standards: The five Cs of HTS: consent; confidentiality; counseling; connections to care, treatment and other services; and correct results [7].

Under the Zimbabwe HTS 2013-2015 strategy, the programme was aimed at increasing the number of people that know their HIV status. Specifically; the 
goals were to increase the percentage of the population who know their HIV status from 57\% for women and 36\% for men in 2011 to $85 \%$ for men and women by 2015. In addition the strategy aimed to increase the percentage of individuals tested for HIV (disaggregated by sero-status and including sero-discordant couples) who link to and enroll into prevention, treatment and care services to $85 \%$ by 2015 [8]. The National HIV Testing Services Strategy expired in December 2015, and in January 2016, the country embarked on an exercise to revise the strategy, considering achievements to. Among the objectives in the new strategy were to align national HTS policies and approaches to the recommended strategies outlined in the updated WHO HTS Guidelines for 2015 and to align testing objectives to the global 90-90-90 fast track targets to ensure Zimbabwe reaches treatment coverage of $81 \%$ of people living with HIV on anti-retroviral treatment by 2020 [9].

HTS is done at the facility or community level. The data is collected and entered into the paper-based HTS register. This information is then collated onto the monthly return form at the district level. This is then uploaded to the Zimbabwe Health Information System (DHIS) electronically and then compiled at the provincial level as Core Output Indicators. Information collected on HTS includes core output indicators on individual clients tested, re-tested, pre-test and post-test counseling, received their results, tested positive, and those referred for post-test support services. More recent indicators include newly tested couples, couples retested, couples who received their results, couples who tested positive and couples referred to post-test support services. Variables are age and sex.

In Zimbabwe, 79.6\% of women knew their HIV status compared to $62.4 \%$ of men in 2015 [10]. In 2015, over 2.2 million tests were done and only $16 \%$ of those were positive, as compared to $22.7 \%$ in 2011 . The National HTS targets from January to June 2016 were to test $1,110,800$ people of which only $54 \%$ was achieved [11]. All provinces missed their targets ranging from 43\% (Bulawayo) to $61 \%$ (Mashonaland East). The current Global Fund HIV grant rating in Zimbabwe went down from A2 to B1 in early 2016 attributed among other reasons to failed HTS targets which dropped by $20 \%$. It was necessary to analyse trends of HTS in Zimbabwe to determine which populations have highest yield and which are in need of special attention to better focus strategies. These findings would guide recommendations towards achieving targets of the "Getting to Zero" by 2030 strategy which aim to have $90 \%$ of people living with HIV know their status by 2020 [12].

\section{Methods}

A secondary data analysis was conducted. The data sources were HTS core output indicators. The data was imported from national data base which captures national core output indicators. Data was extracted manually from the Core Output Indicators from National AIDS Council Head Office found in the National Annual Reports and Excel Sheets. The indicators from different years were merged to create comprehensive indicators to meet objectives of the data set 
analysis. Indicators analyzed included clients pre-test and post-test counseled and clients referred for medical care and psychosocial support. Analyzed were also clients HIV tested, clients tested HIV positive as well as repeat and new tests for HIV. The same indicators were also analyzed amongst couples. Microsoft Office Excel 2007 was used to generate bar graphs and linear graphs to demonstrate the various trends in HTS in the past ten years as well as to calculate the HIV positivity. Statistical significance testing (chi-square for linear trend-Extended Mantel-Haenszel and p-value) was carried out in Epi-Info 7. The projections up to 2020 were calculated in Pandas datatime 64 [ns] class libraries using Time Series Modelling in R. These projections will compare the actual estimates, assuming HTS strategies remain the same, and the ideal achievements if the targets of the first 90 are to be reached.

Permission to carry out the study was sought from the Institutional review board National AIDS Council. Confidentiality was maintained by sharing the results with relevant people and no names were used in the analysis.

\section{Results}

The Core Output Indicators from NAC National Annual Excel Sheets for HTS had a total of 10,847,223 entries. The data set was checked for duplications and completeness of all entries before being analysed. All entries were completely filled and were included in the analysis. A total of 10,847,223 HIV tested clients were captured from the period of 2007 to 2016. Completeness on all variables was $100 \%$

\subsection{Trends in HIV Tests Performed in Zimbabwe from 2007 to 2016}

The total number of HIV tests performed in Zimbabwe has steadily increased over the years from 340,705 in 2007 to $1,974,795$ in 2015. ( $\chi^{2} 0.10492$, p-value 0.74615). Of particular mention is the sharp increase after 2013 where HIV tests increased by $44 \%$ percent from 2013 to 2014 . The observed trend of total number of HIV tests was not statistically significant. While HIV testing has steadily increased over the years the positivity yield rate has decreased. In 2007, 31\% ( $\mathrm{n}=$ $106,884)$ of clients tested were HIV positive whereas only $7 \%(n=121,196)$ of those tested in 2016 have been found HIV positive ( $\chi^{2} 0.01166$, p-value 0.91402 ) (Figure 1).

\subsection{Trends in National HIV Testing by Age and Sex}

HIV testing has steadily increased among all age groups over the ten year period. The 25 - 49 year age-group ( $\chi^{2} 0.0558$, p-value 0.813 ) has tested significantly more than the other age groups throughout the period followed by the $15-24$ year age-group ( $\chi^{2}$ 0.1199, p-value 0.729). Between 2007 and 2013 the over 50 year age-group ( $\chi^{2} 0.0205$, p-value 0.886$)$ had more HIV tests than the under 15 year age-group ( $\chi^{2}$ 0.2966, p-value 0.586) but after 2013 the reverse happened. More under 15 years received HIV tests than over 50 year age-group between 


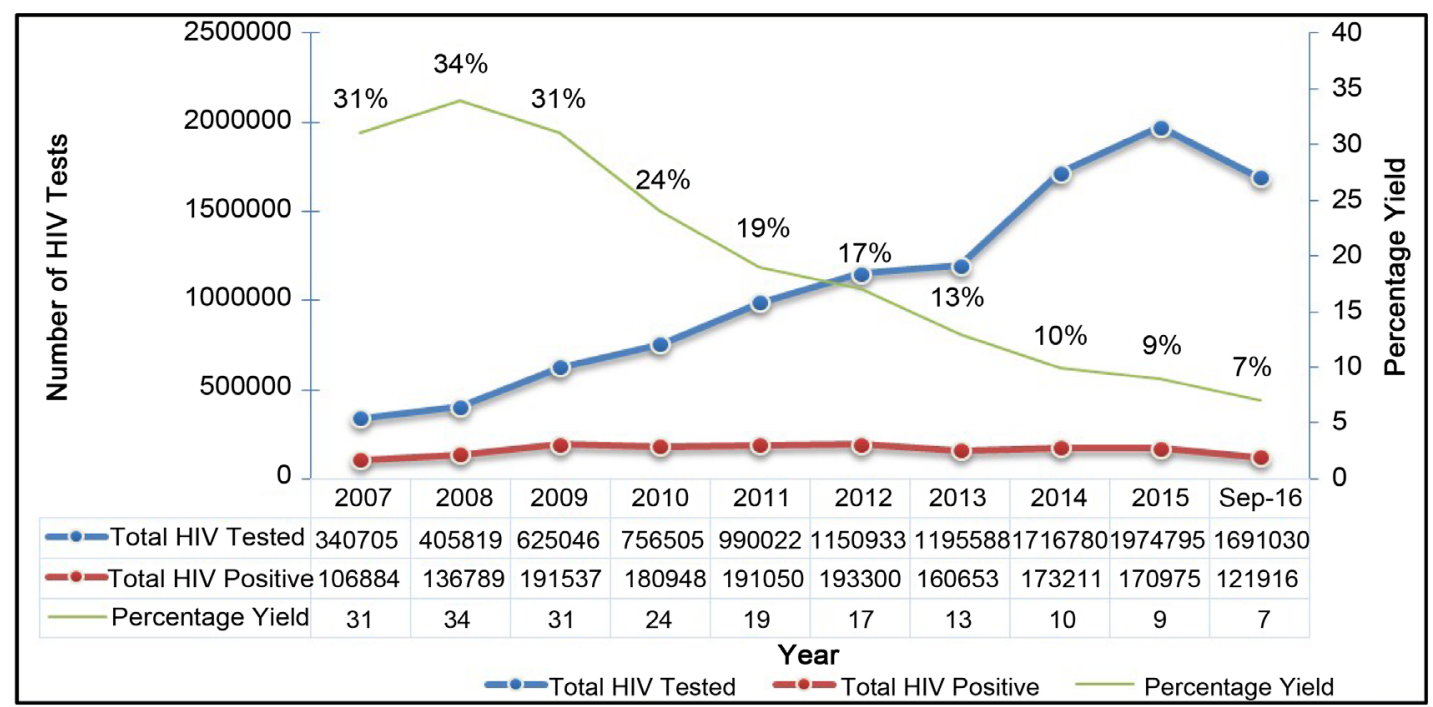

Figure 1. Trends in national total number of HIV tests and total number positive from 2007-2016. $\left(\mathrm{x}_{\text {total HIV Tests }}^{2}=\right.$ $0.10492, \mathrm{p}$-value $=0.74615 ; \quad \mathrm{x}_{\text {total HIV Positive }}^{2}=0.01166, \mathrm{p}$-value $\left.=0.91402\right)$.

2013 and 2016.

Females tested were 5,340,629 compared to 3,612,953 males between 2007 and 2016. The difference in female HIV testing compared to male testing increased gradually over the same time period. In 2007 199,553 females tested which has multiplied ten-fold in 2015 to 1,234,788. ( $\chi^{2}$ 0.1074, p-value 0.743) In 2007, 146,629 males had an HIV test which rose gradually by approximately 6 -fold to 689,622 in 2015 ( $\chi^{2} 0.0614$, p-value 0.804).

\subsection{Trends in HIV Positivity Yield Rate}

Overall yield has decreased in all age-groups in the ten year period. The 25 - 49 year age-group $\left(\chi^{2} 60.94\right.$, p-value $\left.<0.05\right)$ has the highest yield in comparison to all other age-groups with $41 \%$ to 2007 to $11 \%$ in 2016 . The above 50 year age-group $\left(\chi^{2} 42.13\right.$, p-value $\left.<0.05\right)$ peaks in 2008 with a yield of $43 \%$ and is frequently second in highest yield rate. The $15-24$ year age-group $\left(\chi^{2} 80.57\right.$, pvalue $<0.05$ ) has the lowest yield rate with $14 \%$ in 2007 and $5 \%$ in 2016. (Figure 2)

Females $\left(\chi^{2} 43.83\right.$, p-value $\left.<0.05\right)$ have had a higher HIV yield rate than males $\left(\chi^{2} 31.09\right.$, p-value $\left.<0.05\right)$ during ten years but this trend seems to be changing in the last two years. In 2014 and 2015 males and females had the same positivity yield rate of $11 \%$ and $9 \%$ respectively. Up until September 2016 males have had a higher yield rate of $8 \%$ while females have a rate of $7 \%$.

\subsection{Trends in Pre-Test and Post-Test Counseling}

Counselling for HIV testing has risen steadily from 2007 to 2016 which is consistent with general increase in HIV testing. The national trends of HIV counselling show that pre-test counselling ( $\chi^{2} 0.1049, \mathrm{p}$-value 0.746$)$ is consistently higher than post-test counselling $\left(\chi^{2} 0.1135, \mathrm{p}\right.$-value 0.736$)$ throughout the period. 


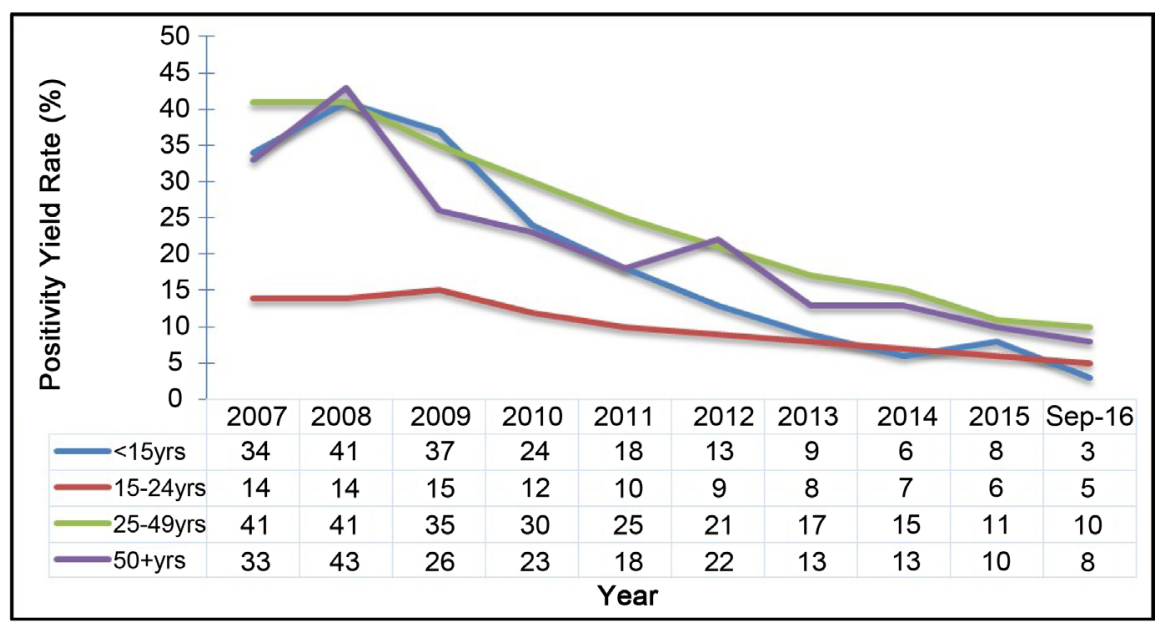

Figure 2. Trends in national HIV positivity yield rate by age from $2007-2016$. $\left(\mathrm{x}_{<15 y r s}^{2}=\right.$ $80.57 \mathrm{p}$-value $<0.05 ; \mathrm{x}^{2}{ }_{15-24 \mathrm{yrs}}=8.764 \mathrm{p}$-value $=0.00307 ; \mathrm{x}^{2}{ }_{25-49 \mathrm{yrs}}=60.94 \mathrm{p}$-value $<0.05$; $\mathrm{x}^{2}{ }_{50+\mathrm{yrs}}=42.13 \mathrm{p}$-value $\left.<0.05\right)$.

\subsection{Trends of Clients Referred for Post-Test Support Services}

Although the number of HIV positive people $\left(\chi^{2} 0.0116\right.$, p-value 0.914$)$ remained approximately constant from 106,884 in 2007 to 121,916 in 2016, the numbers of clients referred for post-services increased over time. Between 2007 and 2009 total referrals per year were lower than total number of clients testing positive per year although there was a slight increase of 82,784 referrals to 160,975. Between 2010 and 2013, post-test services was not used as an indicator in the data set. There was a sharp increase in 2014 to 624,861 referrals where almost 5 times more referrals for post-test services were made than the clients who tested HIV positive $(173,211)$.

\subsection{Trends in New and Repeat HIV Tests}

Repeat tests increased exponentially from $9 \%$ in 2012 to $78 \%$ in 2016 . This indicator was not recorded in 2010 and 2011 (Figure 3).

\subsection{Trends in Individual vs. Couple HIV Testing Uptake and Positivity 2014-2016}

Individuals $\left(\chi^{2} 0.050, \mathrm{p}\right.$-value 0.822$)$ have tested significantly more than couples $\left(\chi^{2}\right.$ 02.472, p-value 0.116) between 2014 and 2016 however couples positive yield rate $\left(\chi^{2} 0.658, \mathrm{p}\right.$-value 0.417$)$ has been greater than individuals' positive yield rate $\left(\chi^{2} 0.23\right.$-value 0.268$)$. In 2014 couples had a yield of $12 \%$ whereas individuals' yield was $11 \%$ (Figure 4 ).

\subsection{Projections of HTS Uptake Till 2020 by Using Time Series Modelling in $\mathbf{R}$}

The linear trend time series projection shows that the actual yearly projected achievements of HIV positive tests will remain almost consistent with previous years ranging from 193,612 to 179,339 , reaching a cumulative total of $1,090,764$ 


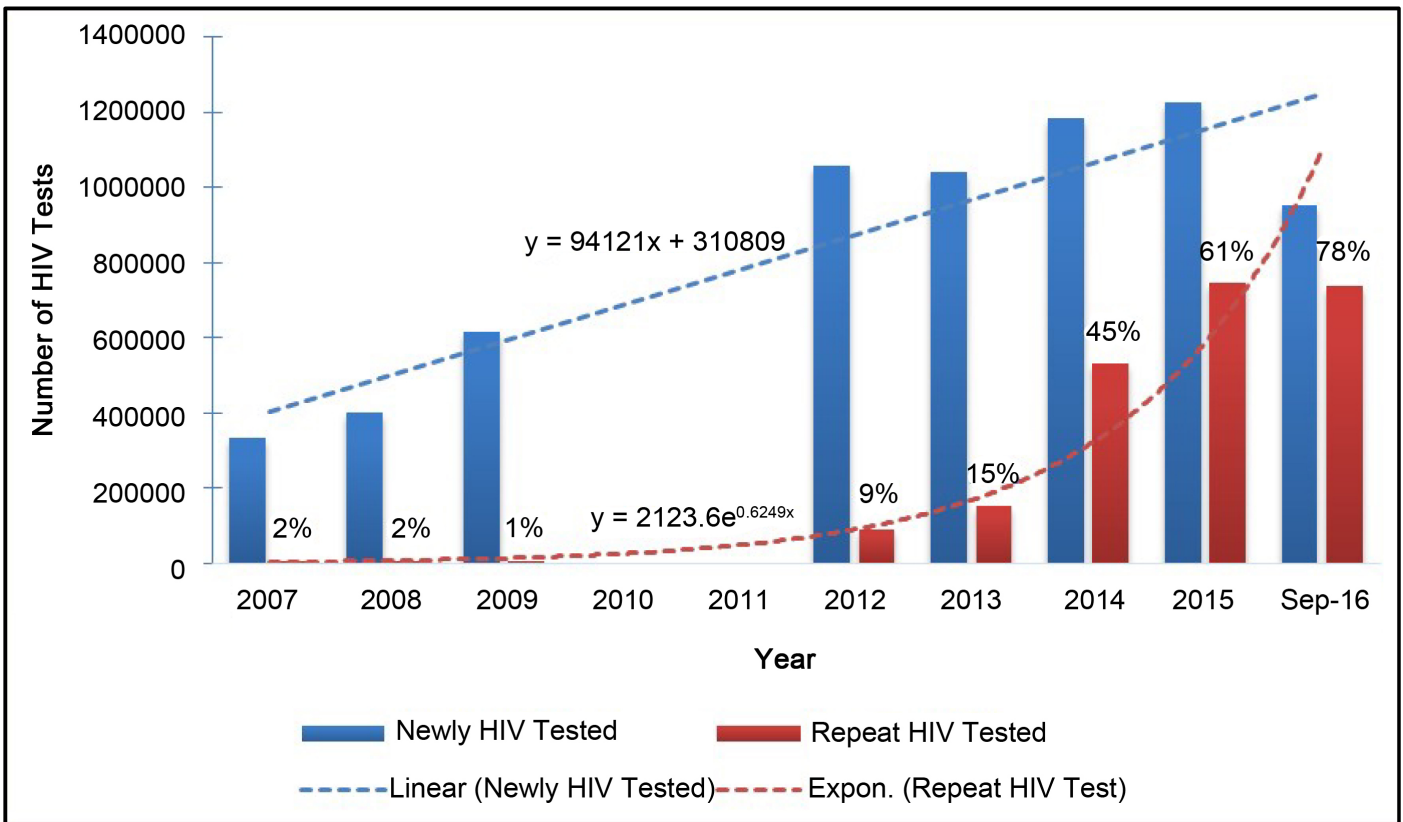

Figure 3. Trends in national newly tested clients and repeat tests from 2007-2016.

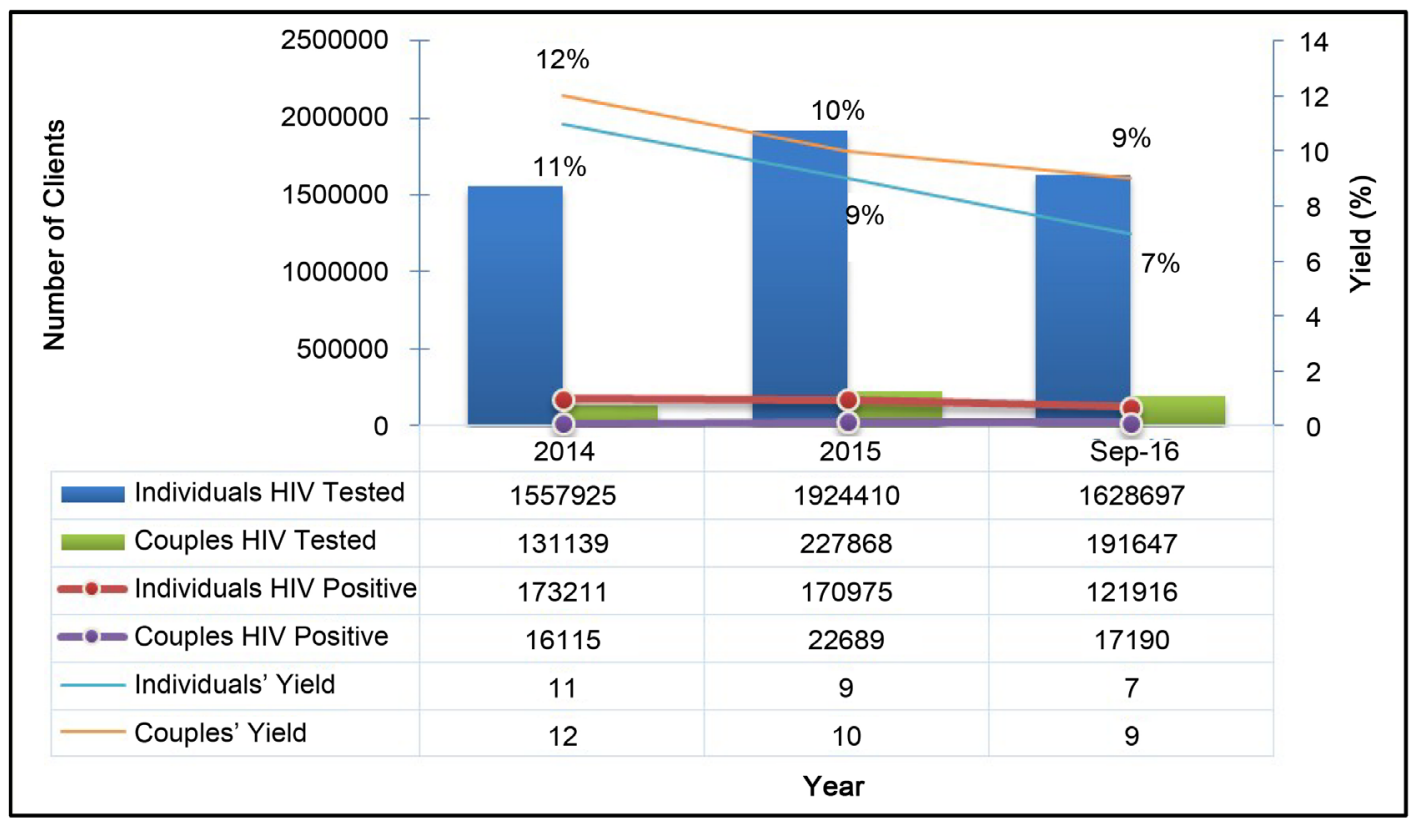

Figure 4. Trends in national individual vs. couple HIV testing and yield in Zimbabwe 2014-2016. $\left(\mathrm{x}^{2}\right.$ indv tested $=$ $0.050 \mathrm{p}$-value $=0.822 ; \mathrm{x}_{\text {couples tested }}^{2} 02.472 \mathrm{p}$-value $=0.116 ; \mathrm{x}_{\text {invd positive }}^{2}=1.23 \mathrm{p}$-value $=0.268 ; \mathrm{x}_{\text {couples positive }}^{2}=$ $0.658 \mathrm{p}$-value $=0.417$ ).

HIV positive tests in 2020. The ideal target would be to have $1,260,000$ people testing positive in 2020 and this would achieve the first 90 . The actual achievement will have fallen short by 169,236 positive tests (Figure 5).

\section{Discussion}

The total number of HIV tests performed in Zimbabwe has steadily increased 


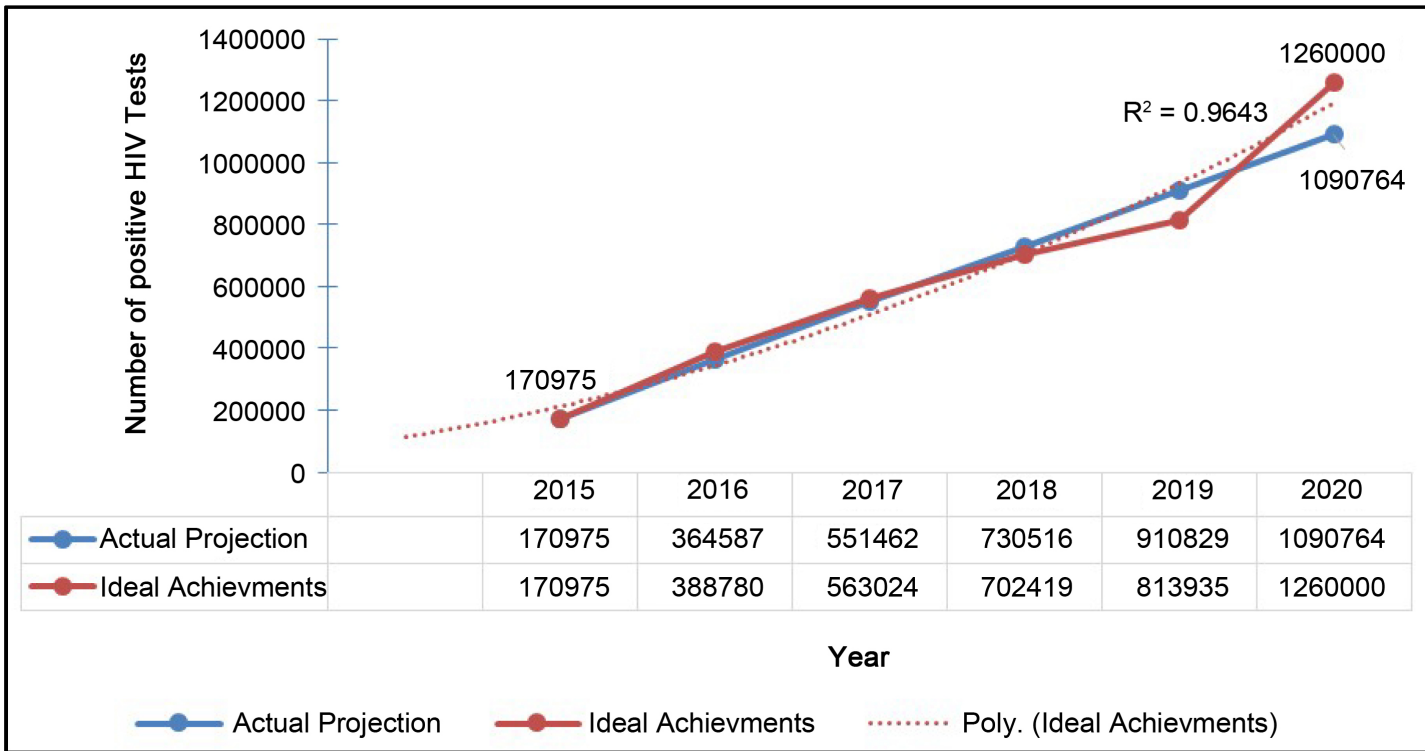

Figure 5. Projections of HTS achievements up to 2020 comparing the actual projection and the ideal projection.

over the ten year period. There is a sharp increase after 2013 where HIV tests increased by $44 \%$ percent from 2013 to 2014 which could be attributed to the roll-out of the Zimbabwe HTS 2013-2015 strategy which aimed at increasing the number of people that know their HIV status. Globally, current HTS approaches have substantially increased the numbers of tests being performed. Data from 108 countries show that in 2010 more than 79 million people received HTS, up from 67.4 million reported in 100 countries in 2009 [7]. In 2014, approximately 150 million children and adults in 129 low- and middle-income countries received HIV testing services. Since 1998, the MEASURE DHS Project's Demographic and Health Surveys (DHS) and AIDS Indicator Surveys (AIS) have collected nationally representative data from women and men from 47 surveys in 29 sub-Saharan countries between 2003 and 2011 about whether they have ever been tested for HIV and whether they received the test results. Sub-Saharan Africa, the world region most affected by HIV, has experienced dramatic increases in the uptake of HIV testing over the past decade. The development of HIV treatment has spurred many changes, including changes in testing strategies, increases in funding for testing, improvements in testing technology, and increased access to testing and treatment [3].

While universal access to HIV testing is a policy priority, in the treatment scale-up era it is particularly important that testing reach HIV-positive persons so that they may obtain care and support. Significant progress in HIV prevention has been made. Globally, new HIV infections declined by 17\% between 2001 and 2008 [13]. The past 10 years have brought some remarkable successes in HIV prevention [14]. In Swaziland the HIV positivity rate is declining among people tested for HIV. In 2012, the positivity rate was at $14 \%$, showing a reduction of almost $3 \%$ when compared with the 2011 positivity rate (16.7\%) [15]. Incidence rates fell more than $25 \%$ in 33 countries, including 22 countries in 
sub-Saharan Africa that carry the highest burden. In Zimbabwe HIV positivity yields have declined steadily over time, requiring more and more tests to identify PLHIV from 22.7 percent to 8.6 percent from 2010 to 2015 [11].

The trend analysis in national HIV testing uptake by age shows that HIV testing has steadily increased among all age groups between 2007 and 2016. Over the years, efforts to test the age groups $2-14$ years and 50+ years have been very weak [15]. Emphasis of youth and adolescent prevention programmes including HTS in the 2013-2016 HTS National Strategy may have been the reason more under 15 years received HIV tests compared to over 50 year age-group between 2013 and 2016. ZIMSTAT Report of ZDHIS 2010/2011 found that in Zimbabwe adults ( 25 - 49 years) accounted for the bulk of HIV rapid tests $(688,808$ in 2014 to 397,039 in 2015) [11]. In some countries with relatively high uptake of HIV testing, including Burundi 2010, Namibia 2006-07, and Swaziland 2006-07, uptake among teens was particularly low, at less than 20 percent. While many factors may affect this age pattern, one of the most important may be that the age of consent for medical treatment is 18 or 21 in these three countries [16]. In other countries with high testing uptake among young people age 15 - 19, laws are much more permissive about testing among adolescents [3].

In Zimbabwe females are consistently getting tested for HIV more than their male counterparts though both sexes have increased their number of HIV tests between 2007 and 2016. The difference in female HIV testing compared to male testing has increased gradually over the same time period. Because of differences in health care seeking behaviour and women's access to reproductive health care, women generally test at a higher rate than do men. Successes of the PMTCT programme also mean more women are accessing HIV testing services. These findings are similar to previous statistics from a study by Takarinda et al. Factors Associated with Ever Being HIV-Tested in Zimbabwe: An Extended Analysis of the Zimbabwe Demographic and Health Survey (2010-2011). They found that in Zimbabwe, HTS in women Ever Tested and Received results increased from 2010 to 2015 from 57.4 percent to 79.6 percent among the 15 - 49 age group whilst percentage in men increased from 35.9 percent to 62.5 percent in the same time period [17]. Disparities in uptake between women and men persist. Findings are also similar in sub-Saharan Africa. In the most recent surveys from 22 of 28 sub-Saharan countries, men are less likely than women to have ever been tested for HIV. Disparities in recent testing (within the past 12 months) are lower than overall disparities between women and men in testing, which may signal that this pattern is changing [3]. The proportion of women ever tested for HIV ranges from 1 percent in Chad 2004 to 76 percent in Rwanda 2010, and for men ranges from 4 to 69 percent, also in Chad 2004 and Rwanda 2010, respectively. The median national uptake of HIV testing for women was 28.8 percent and for men was 17.2 percent [3].

Adults (25 - 49 years) accounted for the bulk of HIV tests and have the highest yield in comparison to all other age-groups. Of interest is the above 50 year 
age-group that is frequently second in highest yield rate. This age-group is often ignored in HIV prevention programmes yet from the data analysis it is evident that there is a high positivity yield which should be taken advantage of.

Females have had a higher HIV yield rate than males during the last ten years but this trend seems to be changing in the last two years with males having higher yield in 2016. HTS campaigns need to focus on reaching male populations.

HIV Test Counselling has risen steadily from 2007 to 2016 which is consistent with general increase in HIV testing. The national trends of HIV counselling show that pre-test counselling is consistently higher than post-test counselling throughout the study period. The disparities in the two are of concern because it reflects a break in the link to care. Although the post-HIV test counselling interview is separate from the pre-test counselling interview, it is linked to it. The pre-test counselling interview should have given the client a glimpse of what to expect in post-test counselling. Pre- and post-test counselling should preferably be done by the same person because the established relationship between the client and counsellor provides a sense of continuity for the client. Post-test counselling links the patient to post-test referral services.

Although the number of HIV positive people remained approximately constant from 2007 to 2016, the numbers of clients referred for post-services increased over time. Between 2007 and 2009, there was a slight increase of 82,784 referrals to 160,975 , which was lower than the total clients tested HIV positive. There was a sharp increase in 2014 to 624,861 referrals where almost 5 times more referrals for post-test services were made than the clients who tested HIV positive. This is because HIV negative clients were now also being referred for post-test services. Referral to care and treatment or to prevention services is key in HTS. All HTS clients should be referred to relevant services after an HIV test. For HIV positive clients, it is mandatory that all clients are referred to Pre-ART services and all HIV negative clients receive prevention services, including male circumcision for men. The HTS provider is responsible for ensuring that referral forms reach the referral facility. In Swaziland during 2012, the HTS card was used in community sites and clients were traced with an average linkage rate of 56 per cent [15]. It was found that in Zimbabwe, 5\% of positives don't get referred to post-test services suggesting a need to monitor providers' compliance with treatment and care guidelines [11].

Repeat testing has risen significantly between 2012 and 2016 which could be a reflection of exhaustion of particular HTS strategies. Up to $78 \%$ of clients tested in 2016 were repeat tests as compared to only $2 \%$ in 2007 . This is similar to a community-based intervention to increase HIV testing and case detection in people aged 16 - 32 years in Tanzania, Zimbabwe, and Thailand repeat HIV testing in community-based VCT communities increased in all sites to reach $28 \%$ of all those testing for HIV by the end of the intervention period compared to standard clinic-based VCT [18]. In Swaziland about 33\% of the tests per- 
formed were repeat tests and of these, $26 \%$ were among males and 39\% were among females.

Individuals have tested significantly more than couples between 2014 and 2016 however couples positive yield has been greater than individuals' positive yield. In 2014 couples had a positivity yield of $12 \%$ whereas individuals' positivity yield was $11 \%$. Most people test alone because of confidentiality emphasized and disclosure often not actively supported. According to an extended analysis of ZDHS 2010/2011 of Ever Being HIV-Tested in Zimbabwe it was found that women and men who were currently or formerly in union were more likely to have ever been tested in comparison to those who were never in union [17]. In Zimbabwe individuals consistently have a higher uptake of testing than couples, but a lower yield rate. There is potential to identify more PLHIV by targeting couples. From January to December 2015 yields in couples were 9.5 percent whilst individuals yielded 8.5 percent positivity yield [11].

Achieving $90 \%$ of HIV positive people knowing their status by 2020 in Zimbabwe is not feasible according to projections made. An increase of approximately 43,000 positive tests per year for the next four years are necessary in order to reach the targets. This is contrary to evidence from a study in Zambia and South Africa reported in February 2016. PopART, also known as HPTN 071, is a large community-randomized trial being carried out in Zambia and Kwazulu Natal, South Africa. The study is testing the impact on HIV incidence of household-based HIV testing and linkage to care by community HIV care providers (CHiPs), and immediate initiation of antiretroviral treatment delivered through routine health care services. Evidence from the pilot phase suggests that although progress is a little slower than expected, it is possible to achieve high levels of testing and treatment initiation - the first two $90 \mathrm{~s}$-in sub-Saharan Africa. Early findings from the PopART study of the impact of a test-and-treat strategy on antiretroviral treatment coverage and HIV incidence show that after one round of household-based testing, linkage to care and offer of immediate antiretroviral treatment, $90 \%$ of adults knew their HIV status and $71 \%$ of adults diagnosed with HIV were on treatment.

\section{Study Limitations}

- The analysis was limited to the variables in the data set. Other variables which could have been helpful to analyze include facility type where testing was done and entry point of HTS.

- Over the 10 year analysis indicators changed several times and in some years were omitted altogether then reintroduced later.

\section{Conclusion}

The total number of HIV tests performed in Zimbabwe has steadily increased in the last ten years. Positivity yield rate has been steadily decreasing between 2007 and 2016. The adult population (25 - 49 year age-group) has had the highest 
number of HIV tests performed as well as the highest yield. Although women test at higher rates than men, males have had higher positivity yield rates in the last two years. There is a consistent disparity between pre-testing and post-testing counselling throughout the ten year period. Most people test alone but there are higher yields amongst couples tested. Repeat testing has increased exponentially in the last five years. According to estimates, Zimbabwe will not achieve the first 90 by 2020 unless approaches towards HTS change.

\section{Recommendations}

As a result of the study some recommendations were made. The data set must in future reports, include facility-type, community and entry point of HTS. This would better inform targeting areas of high yield. Programme evaluation of the current HTS programme must be performed. Discussing successes and challenges of the programme would identify strategies that should be continued or discontinued. This would realign the programme in order to achieve national objectives moving forward. Post-test counseling is consistently lower than pretest counseling which suggests loss-to-follow-up in HTS clients. Facility and community-level HTS referral and linkage systems must be strengthened for appropriate follow-up prevention, treatment and care services by having "onestop shop" facilities that offer testing and treatment. Repeat tests are on the increase suggesting exhaustion of particular strategies. Expansion of coverage of integrated HIV testing and counselling services through implementation of a variety of HIV testing and counselling models is recommended. Examples of these are innovations such as HIV Self-test, index contact testing and moonlight testing. The HTS Strategy 2016-2020 must be finalized and disseminated.

\section{Competing Interests}

The authors declare that they have no competing interests.

\section{Authors' Contributions}

SD: conception, design, acquisition, analysis and interpretation of data and drafting the manuscript. TM: conception, design, acquisition, analysis and interpretation of data and drafting the manuscript. NG: conception, design, data collection, analysis, interpretation and reviewing of several drafts of the manuscript for important intellectual content. TJ: conception, design, data collection, analysis, interpretation and reviewing of several drafts of the manuscript for important intellectual content. MT: conception, design, data collection, analysis, interpretation and reviewing of several drafts of the manuscript for important intellectual content. All authors read and approved the final manuscript.

\section{References}

[1] Denison, J., O’Reilly, K., Schmid, G., Kennedy, C. and Sweat, M. (2007) HIV Voluntary Counseling and Testing and Behavioral Risk Reduction in Developing Coun- 
tries: A Meta-Analysis, 1990-2005. AIDS and Behavior, 12, 363-373. https://doi.org/10.1007/s10461-007-9349-x

[2] Cohen, M., Chen, Y. and McCauley, M. (2011) Prevention of HIV-1 Infection with Early Antiretroviral Therapy. New England Journal of Medicine, 365, 493-505. https://doi.org/10.1056/NEJMoa1105243

[3] Measure DHS Programme (2016). https://dhsprogram.com/pubs/pdf/CR30/CR30.pdf

[4] National AIDS and STI Control Programme, Ministry of Health, Kenya (2015) The Kenya HIV Testing Services Guidelines. NASCOP, Nairobi.

[5] MSMGF's Technical Brief, HIV Testing and Counseling (HTC) (2012) Treatment 2.0, Global HIV Prevention and Treatment Goals. Technical Bulletin Series.

[6] WHO (2016) Statement on HIV Testing and Counseling: WHO, UNAIDS Re-Affirm Opposition to Mandatory HIV Testing.

http://www.who.int/hiv/events/2012/world_aids_day/hiv_testing_counselling/en/

[7] WHO (2012) Service Delivery Approaches to HIV Testing and Counselling (HTC): A Strategic HTC Programme Framework, HIV/AIDS Programme. World Health Organisation.

[8] Ministry of Health and Child Care. National HIV Testing Services Strategy, Zimbabwe, 2011-2015.

[9] Ncube, G. (2016) HIV Testing Services Strategy Revision and Development of 20162020 Strategy, MOHCC.

[10] HIV Estimates (2015).

[11] Zimbabwe National Statistics Agency (ZIMSTAT) and ICF International (2016) Zimbabwe Demographic and Health Survey 2015. Report, Zimbabwe, 8.

[12] UNAIDS (2010) Joint United Nations Programme on HIV/AIDS; Getting to Zero 2011-2015 strategy.

[13] UNAIDS (2009) AIDS Epidemic Update/Publications, Geneva.

[14] Piot, P., Bartos, M., Larson, H., Zewdie, D. and Mane, P. (2008) Coming to Terms with Complexity: A Call to Action for HIV Prevention. The Lancet, 372, 845-859.

[15] Kingdom of Swaziland, Ministry of Health (2012) HTC Programme, Annual Report, Strategic Information Department.

[16] UNDPH, AIDS Group (2012) Global Commission on HIV and the Law: Risk, Rights and Health. UNDP, New York.

[17] Takarinda, K., Madyira, L., Mhangara, M., Makaza, V., Maphosa-Mutsaka, M., Rusakaniko, S., et al. (2016) Factors Associated with Ever Being HIV-Tested in Zimbabwe: An Extended Analysis of the Zimbabwe Demographic and Health Survey (2010-2011). PLoS ONE, 11, e0147828. https://doi.org/10.1371/journal.pone.0147828

[18] Sweat, M., Morin, S., Celentano, D., Mulawa, M., Singh, B., Mbwambo, J., et al. (2011) Community-Based Intervention to Increase HIV Testing and Case Detection in People Aged 16-32 Years in Tanzania, Zimbabwe, and Thailand (NIMH Project Accept, HPTN 043): A Randomised Study. The Lancet Infectious Diseases, 11, 525532. 
Submit or recommend next manuscript to SCIRP and we will provide best service for you:

Accepting pre-submission inquiries through Email, Facebook, LinkedIn, Twitter, etc. A wide selection of journals (inclusive of 9 subjects, more than 200 journals)

Providing 24-hour high-quality service

User-friendly online submission system

Fair and swift peer-review system

Efficient typesetting and proofreading procedure

Display of the result of downloads and visits, as well as the number of cited articles Maximum dissemination of your research work

Submit your manuscript at: http://papersubmission.scirp.org/

Or contact ojepi@scirp.org 